\title{
Morning and Evening People: Examination of an Identifying Scale and of Social- and Self-Perceptions of Personality Differences
}

\author{
Christine Hepburn \\ Pitzer College \\ Vilma ORTIZ \\ University of Michigan
}

AND

ANNE LOCKSLEY

New York University

\begin{abstract}
An experiment was conducted aimed first at establishing the reliability and validity of a measure of individual differences in sleeping schedules. Two samples of subjects completed a questionnaire concerning their sleeping behavior which was scored according to the diurnal or nocturnal pattern of their responses. The scores were then used to classify subjects as morning people or evening peoplc. Both the internal reliability and cross-time reliability of the scale were assessed and found to be high. In addition, three indices of construct validity, obtained 4 months after the administration of the scale, supported its validity. In a second part of the experiment, subjects classified as either morning or evening people rated themselves on a set of behavioral and personality dimensions. Another sample of subjects indicated their perceptions of morning people and evening people in general, along the same dimensions. It was found that subjects did have strong beliefs about personality and behavioral differences between morning people and evening people. However, the self-perceptions of the actual morning and evening people did not differ, thereby casting doubt on the validity of popular stereotypic perceptions about the two groups.
\end{abstract}

It is commonly believed that there are night people and morning people, that is, that stable individual differences in sleeping and waking times

This research was supported by NSF Grant BNS-7912940 to Anne Locksley. The authors are grateful to Roy Neuner and Gary Brill for their assistance with various aspects of this research, and to Norma J. Miller for preparation of the manuscript. Requests for reprints should be sent to Dr. Christine Hepburn, Department of Psychology, Pitzer College, Claremont, CA 91711. 
exist, and furthermore, that these differences are related to a wide range of personality variables. These popular beliefs are addressed by the present research which was aimed at assessing people's stereotypic beliefs about morning and evening people. In addition, the present research was concerned with actual morning and evening people's perceptions of their own characteristics, and thus with obtaining at least preliminary evidence regarding the validity of popular stereotypic perceptions of morning versus evening people.

Given the latter concern, it was necessary to be able to meaningfully identify those in the population who may be considered morning people and those who may be considered evening people. Accordingly, using a recently developed "morningness-eveningness" questionnaire (Horne \& Ostberg, 1976), the present study set out to obtain evidence of reliable. valid individual differences among a normal, college-age sample in the tendency to follow morning or evening sleep schedules. Evidence that the morningness-eveningness questionnaire yields such differences would not only enable the present question regarding the validity of social perceptions of morning and evening people to be pursued, but would enable other investigators to confidently employ this measure in studying other issues related to people's sleeping/waking schedules. Given the current trend toward flexitime work schedules and night shift work (e.g., Melbin, 1979), research examining the flexibility, long-term stability, and correlates of individual differences in sleeping and waking times would potentially be important. Furthermore, it seems likely that people who would be classified as morning people or evening people may worry needlessly that their inability to fall asleep, or stay asleep, at the times that others do reflects a neurotic personality problem. Indeed the term, "insomniac," is viewed as an indicator of emotional disturbance when in fact it may simply be a consequence of a nocturnal circadian rhythm. If so, remedies designed to "relax" a person may remain ineffective. Thus determining the stability of individual differences in sleeping schedules would be an important first step to an understanding of its course and consequences.

The second part of the present study was conducted to assess people's stereotypic beliefs about the personality characteristics of morning and evening people and to compare these beliefs to self-ratings along the same characteristics by those previously identified as actual morning and evening people. Several studies have investigated relationships between personality characteristics and various types of individual differences in sleeping behavior (e.g., Blake, 1967; Blake \& Corcoran, 1972; Colquhoun, 1960; Hartmann, Baekeland, \& Zwilling, 1972; Horne \& Ostberg, 1977; Webb \& Bonnet, 1978; Webb, Bonnet, \& White, 1976). For example, Webb et al. (1976) obtained correlations between sleep stages, defined 
by the EEG, and personality measures, finding no evidence of significant relationships. Other studies have looked for personality differences between people who regularly sleep for extremely few hours and people who regularly sleep for very many hours, but have yielded mixed results. Hartmann, Baekeland, and Zwilling (1972) report findings that "short" sleepers are more extraverted and less worried than "long" sleepers, but Webb and Friel $(1970 ; 1971)$ did not obtain these differences (see Webb, 1979 for a review). More relevant to the present study is past research examining personality differences among people who go to sleep and wake up early as opposed to late. Evidence linking greater extraversion with the tendency to sleep and wake up later comes from Horne and Ostberg's (1976) finding that evening scorers on their questionnaire have later temperature peak times than morning scorers, coupled with Blake and Corcoran's (1972) finding that extraverts have later peak times than introverts. In addition, Tune (1969) found that people who got up later were more extraverted than people who got up earlier, although this held only for subjects over 40 years of age and was not found for 20- to 29year-olds. Furthermore, when Horne and Ostberg (1977) correlated morningness/eveningness, introversion/extraversion, and temperature peak times, they again obtained the relationship between morningness/eveningness and temperature peak time, but found no significant relationships for introversion/extraversion. Finally, Webb and Bonnet (1978) administered the morningness/eveningness questionnaire, a sleep log, and measures of both introversion and locus of control to 100 college students, and found no differences between morning and evening types on either of the personality measures. Thus, there is presently at best only weak evidence of personality differences between people who tend to go to sleep and wake up early and people who tend to go to sleep and wake up late.

Observations of differences in sleeping behavior, however, certainly appear to lend themselves to psychological theorizing. Some popular beliefs imply that the times at which people go to sleep and get up influence various aspects of their lives or personalities. This is exemplified by the famous quote of Benjamin Franklin, "Early to bed and early to rise makes a man healthy, wealthy, and wise." Other popular beliefs rest on the assumption that people stay up late or go to bed early because of the type of person they are. For example, communists and other subversives may be expected to stay awake long into the night, the better to conceal shady activities from the rest of society. Whether nocturnal or diurnal sleeping patterns are viewed as causes or effects of personality variables, the result is stereotypic beliefs about the probable traits or characteristics of morning people versus evening people. Stereotypic beliefs, by "personality-izing" observed differences between people, may 
well interfere with the ability to recognize and address problems stemming from differences in sleeping patterns. It is therefore necessary to determine the validity of beliefs about associations between personality characteristics and sleeping behavior. As a preliminary step toward this goal, the present study compared social perceptions of morning and evening people along many different characteristics to self-ratings by people identified in the first part of the experiment as actual morning people and evening people.

\section{METHOD}

\section{Subjects}

Three different samples of subjects participated in this experiment. One sample (A) consisted of 260 introductory psychology students, another sample (B) consisted of 60 introductory personality students, and another sample $(C)$ consisted of 60 introductory psychology students, all enrolled at New York University.

\section{Measurement and Classification Procedure}

Horne and Ostberg's (1977) morningness-eveningness questionnaire, or Owt-Lark Scale, was first administered to the 260 subjects in Sample A. 'This questionnaire consists of 26 questions which ask, for example, about the times of day one usually wakes up on weekdays and weekends, scored in such a way as to discriminate responses to each question in terms of diurnal versus nocturnal circadian rhythms. On the basis of interitem correlations and factor analyses of the responses of these subjects, six of the original items were deleted from the scale, resulting in a final version of the questionnaire consisting of 19 items. ${ }^{2}$ This version of the scale was then administered to the 60 subjects in Sample B.

Responses to the 19 items were scored in the nocturnal direction, and a scale score for each subject was obtained by standardizing the responses to each item and averaging each subject's $z$ scores across the set of 19 items. The responses to each item were standardized before combination so that differenzes in the size of the items response scales would not bias the contribution of each item to the final score. Subjects in each sample were classified as a morning person or an evening person if they scored in the upper or lower thirds. respectively, of their distributions. It should be noted, however, that the full range of scores in both samples were used for correlational analyses.

\section{Procedure for Obtaining Estimates of the Internal and Cross-Time Reliability and of the Construct Validity of the Owl-Lark Scale}

The internat reliability for the 19-item version of the scale was estimated by computing Cronbach's coefficient $\alpha$ for Sample $A$ and by cross-validating the estimate with the responses of subjects in Sample B. The scale was administered a second time to subjects in Sample B, exactly 3 weeks after the first administration. Correlating subjects scores at the two time points permitted an estimate of the cross-time reliability of the scale. Construct validity for the scale was assessed in three ways, involving three independent groups of nocturnals and three independent groups of diurnals selected from Sample $A$.

\footnotetext{
1 The subjects came from eight class sections which met at different time periods, from 9:00 AM to $3: 30$ PM.

${ }^{2}$ Copies are available by request to the first author, Pitzer College, Claremont, CA 91711. In addition, results of the factor analyses and a listing of the mean responses from the original sample of 260 subjects, broken down by classification, are available.
} 
First, 4 months after the administration of the scale, 35 diurnals and 33 nocturnals were telephoned at home, on a weekday, between 7:30 and 8:15 AM and asked whether or not they had been wakened by the call. Second, 4 months after the scale administration, 16 diurnals and 15 nocturnals were called during an afternoon of the final exam period, and asked to select a number on a 5-point scale to indicate how often they had stayed up all night during the exam preparation week to study or to write a paper for a course. Third, also 4 months after the scale administration, 15 diurnals and 15 nocturnals were telephoned one afternoon and asked how often they had missed classes which met before noon, throughout the past semester, from "Never" to "Ten times or more." It was first ascertained that the two groups did not differ on the average number of morning classes in which they were enrolled. Validity for the classification procedure would be indicated if subjects classified as nocturnal were more likely to be asleep between 7:30 and 8:15 AM, more likely to have stayed up all night studying while preparing for final exams, and to have missed more classes which met before noon, than subjects classified as diurnal.

\section{Procedure for Obtaining Self-Reports of Attributes from Actual Morning People and Evening People}

A list was constructed of 91 trait (e.g., unconventional, self-controlled, healthy) and behavioral (e.g., smokes cigarettes, engages in regular physical exercise) dimensions which were judged by the authors as possible components of stereotypes of morning and evening people. The 91 dimensions were randomly ordered. Subjects in Sample B were asked to rate the extent to which each characteristic was true of themselves on 7-point scales, from $1=$ not at all true to $7=$ very true. These self-reports were assessed 4 weeks after the second administration of the Owl-Lark Scale. Subjects were led to believe that the two inventories were entirely unrelated, and indeed, during debriefing, no subjects reported suspecting a connection between the two.

\section{Procedure for Assessment of Stereotypic Beliefs about Morning People and Evening People}

Subjects in Sample $C$ were presented with the same list of 91 dimensions used to obtain self-reports of morning people and evening people, and asked to rate the extent to which each characteristic is true of morning people and of evening people in general. The categories of morning and evening people were defined for the subjects solely in terms of individual differences in the times of day people prefer to go to sleep and wake up.

\section{RESULTS}

The estimates of the internal and temporal reliability, and the estimates of the construct validity of the modified version of the Owl-Lark Scale are presented in Tables 1 and 2. It can be seen there that they were indicative of relatively stable individual differences in sleeping behavior. Cronbach's coefficient $\alpha$ remained high when cross-validated on Sample B. The cross-time reliability of the scale, estimated across a 3-week interval, was also quite high. ${ }^{3}$ Furthermore, as Table 2 demonstrates, all

" It should be noted that the computation of the cross-time reliability of the revised Owl-Lark Scale across a 3-week interval is not intended to address the important issue of the long-term stability of the individual differences being measured. Rather, this crosstime correlation was computed to provide standard, psychometric support for the reliability 
TABLE 1

INDICES OF RELIABILITY OF RESPONSES TO THE Modified OWI-Lark Scale

\begin{tabular}{lcc}
\hline $\begin{array}{l}\text { Indices of } \\
\text { response }\end{array}$ & Sample A & Sample B \\
reliability & $(N=260)$ & $(N=45)$ \\
\hline $\begin{array}{l}\text { Coefficient } \alpha \\
\text { Temporal } \\
\quad \text { correlation }\end{array}$ & .75 & .83 \\
\hline
\end{tabular}

Note. The modified version of the Owl-Lark Scale comprises only 19 of the original 26 items (Horne \& Östberg, 1977). Coefficient $\alpha$ for Sample $B$ represents a cross-validation of item selection based on factor analyses of responses by Sample A. The reported temporal correlation was computed over a 3-week interval.

three of the predictions for behavioral differences between nocturnal and diurnal subjects assessed 4 months after the scale administration were confirmed. Thus, the results revealed that the procedures for classifying morning and evening people are reasonably reliable and valid. Accordingly, the self-reports of subjects in Sample B who were classified as morning and evening peoplc were compared to the social perceptions of morning and evening people in general provided by subjects in Sample C.

Of the total number of 91 attributes, only 8 had significantly different $(p<.05)$ rates of endorsement by nocturnals and diurnals, a proportion of significant differences which does not depart significantly from the more than five significant differences one would expect by chance alone. In contrast, ratings of the characteristics of evening people and of morning people differed significantly $(p<.05)$ for 59 of the 91 dimensions. It would appear that people believe that evening people differ systematically from morning people on many more dimensions than are actually the case.

Because of the necessarily different procedures for sampling stereotypic ratings versus self-reports, however, the power for the detection of significant differences turned out to be much greater for comparisons of stereotype ratings than for self-reports of evening people and morning

of scores on the scale. A 3-week interval is generally deemed sufficient to ensure that, at time 2, subjects are not merely recalling their time 1 responses, and thus the high correlation obtained strongly supports the claim that the scale is picking up true variance with respect to the relevant dimension. In addition, it should be emphasized that the significant validity check results were obtained with an interval of 4 months between the scale administration and the validity measures. 
TABLE 2

Indices of the VaLidity of the Modified OWL-LaRk Scale

\begin{tabular}{lccc}
\hline Behavioral dimension & Nocturnals & Diurnals & \\
\hline $\begin{array}{l}\text { Proportion asleep between 7:30 } \\
\text { and 8:15 AM }\end{array}$ & .73 & .40 & $z-2.74^{* *}$ \\
$\begin{array}{l}\text { Number times stayed up all } \\
\text { night to study during exam } \\
\text { week }\end{array}$ & 2.87 & 2.00 & $t(29)=2.06^{*}$ \\
$\begin{array}{l}\text { Number times missed morning } \\
\text { classes during semester be- } \\
\text { cause of oversleeping }\end{array}$ & 3.60 & 1.00 & $t(28)=2.38^{*}$ \\
\hline
\end{tabular}

Note. The modified version of the Owl-Lark Scale comprises only 19 of the original 26 items (Horne \& Östberg, 1977). Subjects were classified as nocturnal or diurnal on the basis of their responses to the scale, administered 4 months prior to assessment of values on the three behavioral dimensions. Three different samples of nocturnal and diurnal subjects were used for the three validity checks.

$* p<.05$.

** $p<.01$.

people (Cohen, 1977). The former involved within-subjects $t$ tests and twice the sample size than obtained for the latter. Accordingly, in order to make a fair comparison, ratings of evening and morning people were sampled randomly from the pool of stereotype ratings to create betweensubjects samples with sizes equal to the comparable samples of actual evening people and morning people. In spite of the reduction of power for the assessment of differences between the stereotype ratings, ratings of evening people and of morning people differed significantly for 38 out of the original 91 dimensions $(p<.05)$, and marginally for another 6 dimensions $(.10<p>.05)$. An idea of the nature of the stereotypic beliefs about morning and evening people is provided by Table 3, which presents a representative selection of 20 traits dimensions and the comparisons for both stereotype and self-ratings.

In sum, the results showed that stereotypic beliefs about differences in the characteristics of morning and evening people are not born out by corresponding differences in self-ratings by actual morning and evening people. Even when the power to estimate differences between two groups was equivalent for both the self-report samples and the stereotypic ratings samples, the proportion of perceived differences between evening people and morning people continued to substantially exceed the proportion of reported differences, which did not deviate significantly from what would be expected by chance alone. And it may also be noted that, when significance levels are disregarded altogether and the direction of the stereotypic ratings is compared to the direction of the self-reports (i.e., 
TABLE 3

Mean Self-Reports and Mean Stereotypic Ratings of Nocturnals and Diurnals

\begin{tabular}{|c|c|c|c|c|c|c|}
\hline \multirow[b]{2}{*}{$\begin{array}{l}\text { Trait } \\
\text { dimensions }\end{array}$} & \multicolumn{2}{|c|}{ Self-reports } & \multicolumn{4}{|c|}{ Stereotypic ratings } \\
\hline & $\begin{array}{l}\text { Nocturnals } \\
(N=15)\end{array}$ & $\begin{array}{l}\text { Diurnals } \\
(N=15)\end{array}$ & $t(28)$ & $\begin{array}{l}\text { Nocturnals } \\
(N=15)\end{array}$ & $\begin{array}{l}\text { Diurnals } \\
(N==15)\end{array}$ & $t(28)$ \\
\hline Dependable & 5.69 & 6.13 & -1.24 & 3.87 & 5.07 & $-2.55^{*}$ \\
\hline Dreamy & 4.38 & 3.47 & 1.53 & 4.73 & 3.60 & $2.15^{*}$ \\
\hline Healthy & 5.94 & 6.13 & -.68 & 3.67 & 4.93 & $-3.73^{* * *}$ \\
\hline Imaginative & 4.81 & 5.13 & -.68 & 5.67 & 3.87 & $2.60^{*}$ \\
\hline Impulsive & 4.06 & 3.87 & .37 & 5.00 & 3.93 & $2.31^{*}$ \\
\hline Individualistic & 5.44 & 5.00 & 1.01 & 5.13 & 3.67 & $2.77^{* * *}$ \\
\hline \multicolumn{7}{|l|}{ Interested in } \\
\hline films & 4.39 & 5.08 & -1.16 & 5.33 & 3.73 & $4.36^{* * *}$ \\
\hline Introverted & 3.75 & 4.27 & -.89 & 3.43 & 4.57 & $-2.16^{*}$ \\
\hline Messy & 3.81 & 3.00 & 1.19 & 4.79 & 3.33 & $2.94^{* *}$ \\
\hline Punctual & 4.50 & 5.60 & -1.76 & 3.60 & 5.40 & $-3.18^{* *}$ \\
\hline Rational & 5.13 & 5.33 & -.49 & 4.00 & 5.13 & $-2.06^{*}$ \\
\hline Realistic & 5.13 & 5.67 & -1.41 & 4.13 & 5.13 & $-2.31^{*}$ \\
\hline Rebellious & 3.94 & 3.47 & .81 & 4.87 & 2.73 & $5.11^{* * *}$ \\
\hline Responsible & 5.75 & 5.67 & .21 & 4.13 & 5.07 & $-2.18^{*}$ \\
\hline Self-controlled & 5.75 & 5.47 & .85 & 3.80 & 5.27 & $-3.80^{* * *}$ \\
\hline Self-disciplined & 5.38 & 5.13 & .51 & 4.07 & 5.87 & $-4.43^{* * *}$ \\
\hline Studious & 4.88 & 4.93 & -.12 & 3.87 & 5.33 & $-3.70^{* * *}$ \\
\hline Unconventional & 4.19 & 4.13 & .10 & 4.92 & 3.58 & $2.37^{*}$ \\
\hline Unpredictable & 3.88 & 3.33 & .86 & 5.27 & 3.40 & $3.73^{* * *}$ \\
\hline Unsystematic & 3.50 & 2.80 & 1.27 & 4.67 & 3.13 & $2.71^{*}$ \\
\hline
\end{tabular}

$$
\begin{aligned}
{ }^{*} p & <.05 \\
{ }^{* *} p & <.01 \\
{ }^{* *} p & <.001
\end{aligned}
$$

are nocturnals rated higher or lower than diurnals as compared to whether nocturnals rate themselves higher or lower than diurnals), the directions differ on 34 out of the 91 possible comparisons. Thus the perceived direction of the difference between nocturnals and diumals is opposite to the reported direction of the difference between nocturnals and diurnals for $38 \%$ of the ratings, a proportion one would expect by chance (i.e., were there no relationship between perceived and reported differences).

Finally, the self- and social ratings may be examined with particular attention to characteristics related to extraversion since some research has suggested that evening people may be more extraverted than morning people (Blake \& Corcoran, 1972, Tune, 1969). Out of nine rated traits judged by the authors to be conceptually related to extraversion, however, only one-popular-was differentially endorsed by evening and morning people, and the difference was opposite in direction to what one would 
expect. That is, evening people rated themselves as less popular than morning people. As far as social perceptions were concerned, evening people in general were rated as more popular, outgoing, and sociable than morning people, $t$ 's $(58) \geqslant 2.16, p$ 's $<.05$, but were not rated as significantly more extraverted, more friendly, or less introverted, unsociable, and unfriendly, $t$ 's $(58) \leqslant 1.35, p$ 's $>.10$. Moreover, evening people were perceived as significantly more lonely than morning people, $t(58)=2.47, p<.05$. Thus, the present self-rating results clearly do not support the hypothesis that evening people are more extraverted than morning people, and, interestingly, this hypothesis is only very weakly reflected in subjects' stereotypic beliefs about the two types of people.

Ratings of characteristics related to anxiety or neurosis were also of particular interest, given the possibility noted in the introduction that evening people may infer that they have personality problems based on their difficulty conforming to a diurnal schedule. None of the six characteristics judged to be conceptually related to anxiety, however, were differentially endorsed by morning and evening people, all $t$ 's $(58) \leqslant 1.36$, $p$ 's $>.10$. There was some evidence, though, that evening people are stereotypically viewed as being more anxious; evening people in general were rated as significantly more anxious, tense, and emotional than morning people in general, $t$ 's $(58) \geqslant 2.20, p$ 's $<.05$, although they were not rated as significantly more neurotic, more maladjusted, or less easygoing, $t$ 's $(58)$ $\leqslant 1.81, p$ 's $>.05$.

\section{DISCUSSION}

The results of this experiment demonstrate that individual differences in sleeping schedules obtain among a normal sample of people. A selfreport method for assessing these individual differences (Horne \& Ostberg, 1977) proved to have impressive internal and temporal reliability. Even more convincingly, classification of subjects on the basis of their responses to the questionnaire reliably predicted differences in relevant behavior observed 4 months later. Nocturnal people were significantly more likely than diurnal people to be asleep when telephoned between $7: 30$ and 8:15 AM. Nocturnal students stayed up all night to study for an exam more often during exam week than diurnal students. And nocturnal students reported missing more morning classes because of oversleeping than did diurnal students. It would seem, then, that individual differences in the time of day one prefers to fall asleep and to wake up are sufficiently stable to be reliably and validly measured using the Owl-Lark questionnaire.

It should be noted that subjects in the present study were college students, a group of people widely acknowledged to be more variable sleepers than older adults. College students are young and therefore are expected to have relatively unstable sleeping patterns, and in addition, 
external constraints may well be more variable in college than in most postcollege settings. Thus, that the present subjects' scores on the OwlLark scale were in fact found to be internally and temporally reliable. and to be predictive of relevant behavior after 4 months, is noteworthy. While the question of whether the differences in sleep schedules among individuals in this study would tend to persist throughout their adult lives remains to be answered, the present results, as well as those of Horne and Ostberg (1977) and Webb and Bonnet (1978). leave little doubt that the Owl-Lark scale does yield reliable and valid individual differences. even among college students.

The results of the assessment of stereotypic beliefs about morning and evening people revealed that it is generally believed that morning and evening people are different with respect to a large number of personality and behavioral characteristics. Inspection of these beliefs suggests that nocturnal people are generally perceived to have more deviant traits than diurnal people. It appears that the lack of conformity implied by a sleeping pattern which deviates from the diurnal "norm" is generalized to a variety of personality and behavioral dimensions. Self-ratings along the same set of dimensions by actual morning and evening people, however, were not found to differ above what would be expected by chance alone. Although self-reports do not provide a definitive criterion for the validity of perceptions of group differences, the very substantial disparity obtained here between popular perceptions and self-perceptions suggests that generalization from sleeping behavior to personality factors may be unfounded.

Again, it must be noted that the present results regarding personality differences between morning and evening people come from a sample of college students. Thus, it remains entirely possible that, as suggested by Tune's (1969) research, personality differences might be found between morning and evening people who have maintained their respective sleep scheduling tendencies over many years, or who developed their different sleep schedules later in life. Of course, it is not at all clear why preferred sleep schedules and personality might be related for older, but not for younger, adults. Indeed, it could be argued that such a relationship should best be detected among college students, since their sleeping preferences may be relatively undetermined by career or family considerations. In any case, the experiment reported here demonstrates the existence of individual differences in morningness/eveningness, unaccompanied by corresponding differences in a wide range of self-reported personality characteristics.

\section{REFERENCES}

Blake, M. J. (1967). Relationships between circadian rhythm of body temperature and introversion-extraversion. Nature (London), 215, 896-897. 
Blake, M. J., \& Corcoran, D. W. (1972). Introversion-extraversion and circadian rhythms. In W. P. Colquhoun (Ed.), Aspects of human efficiency. London: English Univ. Press.

Cohen, J. (1977). Statistical power analysis for the behavioral sciences. New York: Academic Press.

Colquhoun, W. P. (1960). Temperament, inspection efficiency and time of day. Ergonomics, 3, 377-378.

Ilartmann, E., Baekeland, F., \& Zwilling, G. (1972). Psychological differences between long and short sleepers. Archives of General Psychiatry, 26, 463-468.

Horne, J. A., \& Ostberg, O. (1976). A self-assessment questionnaire to determine Morningness-eveningness. Internutional Journal of Chronobiology, 4, 97-110.

Horne, J. A., \& Ostberg, O. (1977). Individual differences in human circadian rhythms. Biological Psychology, 5, 179-190.

Melbin, M. (1979). Settling the frontier of night. Psychology Today, 13, 40-52.

Tune, G. J. (1969). The influence of age and temperament on the adult human sleepwakefulness patterns. British Journal of Psychology, 60, 431-441.

Webb, W. B. (1979). Are short and long sleepers different? Psychological Reports, 44, 259-264.

Webb, W. B., \& Bonnet, H. H. (1978). The sleep of "morning" and "evening"' types. Biological Psychology, 7, 29-35.

Webb, W. B., Bonnet, H. H., \& White, R. M. (1976). State and trait correlates of sleep stages. Psychological Reports, 38, 1181-1182.

Webb, W. B., \& Friel, J. (1970). Characteristics of "natural" long and short sleepers: A preliminary report. Psychological Reports, 27, 63-66.

Webb, W. B., \& Friel, J. (1971). Sleep stage and personality characteristics of "natural" long and short sleepers. Science, 171, 587-588. 